\title{
Tamoxifen-loaded solid lipid nanoparticles-induced apoptosis in breast cancer cell lines
}

\begin{abstract}
An in vitro study was conducted to determine the apoptosis induced by tamoxifen (TAM) and TAM-loaded solid lipid nanoparticles (SLNs) in breast cancer cell lines, MCF-7 and MDAMB231 cells. The effect of free drug and drug-loaded SLN on the cell lines was characterised by cell morphology and cell cycle distribution using phase contrast microscopy, nuclear morphology and flow cytometry, respectively. The results showed that TAM-loaded SLNs have an equally efficient cytotoxic activity against MCF-7 and MDA-MB231 cells, compared to free TAM, and the half maximal inhibitory concentration (IC50) of TAM-loaded SLNs was generally lower than that of free TAM. In the presence of TAM and TAM-loaded SLN, the viability of the both cells diminishes and the cancer cells lose their normal morphological characteristics, detaches, aggregates and later develops apoptotic bodies. Flow cytometry analysis showed that TAM-loaded SLN like the free TAM caused a dose- and timedependent apoptosis without cell cycle arrest of human breast cancer cells. Therefore, TAMloaded SLN has great potential in human medicine for the treatment of breast cancers.
\end{abstract}

Keyword: Apoptosis; Breast cancer cell lines; Solid lipid nanoparticles; Tamoxifen 